\title{
Clinical Study \\ The Frequency of Epidermal Growth Factor Receptor Mutation of Nonsmall Cell Lung Cancer according to the Underlying Pulmonary Diseases
}

\author{
Kazuhiro Usui, ${ }^{1}$ Tomonori Ushijima, ${ }^{2}$ Yoshiaki Tanaka, ${ }^{1}$ Chiharu Tanai, ${ }^{1}$ \\ Hiromichi Noda, ${ }^{1}$ Norifumi Abe, ${ }^{3}$ Hajime Horiuchi, ${ }^{2}$ and Teruo Ishihara ${ }^{1}$ \\ ${ }^{1}$ Division of Respirology, NTT Medical Center Tokyo, 5-9-22 Higashigotanda Shinagawa, Tokyo 141-0022, Japan \\ ${ }^{2}$ Division of Diagnostic Pathology, NTT Medical Center Tokyo, 5-9-22 Higashigotanda Shinagawa, Tokyo 141-0022, Japan \\ ${ }^{3}$ Division of Chest Surgery, NTT Medical Center Tokyo, 5-9-22 Higashigotanda Shinagawa, Tokyo 141-0022, Japan
}

Correspondence should be addressed to Kazuhiro Usui, usui@east.ntt.co.jp

Received 13 August 2011; Revised 29 September 2011; Accepted 29 September 2011

Academic Editor: Charlie Strange

Copyright (C) 2011 Kazuhiro Usui et al. This is an open access article distributed under the Creative Commons Attribution License, which permits unrestricted use, distribution, and reproduction in any medium, provided the original work is properly cited.

\begin{abstract}
Background. Although epidermal growth factor receptor-tyrosine kinase inhibitors (EGFR-TKIs) are effective in patients with nonsmall cell lung cancer with epidermal growth factor receptor (EGFR) mutation, EGFR-TKIs have a risk of inducing fatal interstitial lung disease (ILD). The selection of chemotherapy based on the EGFR mutation status is recommended, however, the frequency of EGFR mutation in patients with ILD and the efficacy and safety of EGFR-TKI in patients with ILD and EGFR mutation are unknown. Methods. We retrospectively reviewed the association of the EGFR mutation status of nonsmall cell lung cancer and pulmonary diseases. Based on high-resolution computed tomography (HRCT) performed at diagnosis of lung cancer, patients were categorized into three groups: normal, emphysema, and fibrosis. Results. Of 198 patients with nonsmall cell lung cancer, we identified $52(26.3 \%)$ patients with an EGFR mutation. EGFR mutations were identified in $43(35.2 \%)$ of 122 patients with normal lungs, 8 (13.6\%) of 59 with emphysema, and $1(5.9 \%)$ of 17 with pulmonary fibrosis. Of the 52 patients with EGFR mutation, 43 patients received gefitinib. One patient with an EGFR mutation and fibrosis developed fatal ILD. There was not a significant difference in median overall survival from gefitinib treatment between never-smokers and smokers (797 days versus not reached; $P=0.96)$. Conclusions. Patients with sensitive EGFR mutation and normal lungs may benefit from an EGFR-TKI treatment even if they have smoking history.
\end{abstract}

\section{Introduction}

Gefitinib is a reversible epidermal growth factor receptor tyrosine kinase inhibitor (EGFR-TKI) used for the treatment of nonsmall cell lung cancer patients [1]. Although demographic and clinical factors such as East-Asian race, female gender, nonsmoking status, and adenocarcinoma were shown to be predictive of the efficacy of gefitinib, two pivotal studies showed that the presence of somatic mutations in the kinase domain of epidermal growth factor receptor (EGFR) strongly correlates with increased responsiveness to EGFRTKIs in patients with nonsmall cell lung cancer $[2,3]$. It was later found that the subgroups of patients with nonsmall cell lung cancer who had sensitivity to gefitinib had a high incidence of EGFR mutations $[4,5]$. Selecting patients on the basis of EGFR mutations, rather than clinical factors, would likely result in a population with a greater sensitivity to gefitinib. First-line gefitinib for patients with advanced nonsmall cell lung cancer who are selected on the basis of EGFR mutations improves progression-free survival, with acceptable toxicity, compared with standard chemotherapy, although it failed to prolong overall survival $[6,7]$.

However, EGFR-TKI increases the risk of developing lifethreatening interstitial lung diseases (ILDs). The estimated incidence of ILD is low in many countries (e.g., $0.3 \%$ in the United States) [8] but is relatively high (4 to 6\%) in Japan $[9,10]$. An older age, poor World Health Organization performance status, reduced normal lung area on computed 
tomography scans, preexisting chronic ILD, and concurrent cardiac diseases are known as risk factors for ILD in gefitinib treatment [10]. Although an assessment of pulmonary comorbidities, especially ILDs, is important to decrease the incidence of ILD induced by chemotherapy, the frequency of EGFR mutation in patients with pulmonary fibrosis and the clinical feature of these patients are not clear.

We reviewed 198 patients who were examined for EGFR mutation status and assessed the association of EGFR mutations with the underlying pulmonary diseases on chest high-resolution computed tomography (HRCT).

\section{Methods}

The medical records of a series of consecutive patients with histologically- or cytologically-proven lung cancer, who were tested for EGFR mutation status in the Division of Diagnostic Pathology, NTT Medical Center Tokyo between April 2008 and November 2010, were retrospectively reviewed. The status of EGFR mutation was examined in a clinical practice, not investigational setting, to decide the indication of EGFR-TKI treatment. Although most patients with severe pulmonary fibrosis or squamous cell carcinoma were excluded from the EGFR mutation test in this period, gender, smoking status, and the existence of emphysema were not considered as the exclusion criteria of the test. Patients with emphysema and fibrosis on chest HRCT at the diagnosis of lung cancer were prospectively identified, and the data before lung cancer treatment was recorded to assess their risk of ILD. Only patients who had a chest HRCT scan, which was performed at diagnosis of lung cancer and was available for review, were included in the study. The study protocol was reviewed and approved by the Ethics Committee of NTT Medical Center Tokyo.

Patients were categorized into three groups; those with normal lungs (except for the tumor), emphysematous lungs, or fibrotic lungs, based on chest CT findings as described previously $[11,12]$. Patients who met the following criteria were categorized as having emphysema: the presence of emphysema on CT, defined as well-demarcated areas of decreased attenuation in comparison with contiguous normal lung, and marginated by a very thin $(<1 \mathrm{~mm})$ wall or no wall, and/or multiple bullae $(>1 \mathrm{~cm})$ with upper zone predominance. Patients who met the following criteria were categorized as having fibrosis: the presence of diffuse parenchymal lung disease with significant pulmonary fibrosis on CT, defined as reticular opacities with peripheral and basal predominance, honeycombing, architectural distortion, and/or traction bronchiectasis or bronchiolectasis; focal groundglass opacities and/or areas of alveolar condensation may be associated, but should not be prominent. Patients who met neither criterion emphysema nor fibrosis were categorized as normal. The electronic medical records were reviewed to obtain clinical and demographic data, including gender, age, smoking history, histology results, clinical stage of lung cancer, treatment, treatment-related toxicities, and survival.

2.1. EGFR Mutation Analysis. The presence of EGFR mutations was determined by the peptide nucleic acid-locked nucleic acid PCR clamp method as described previously [13]. The investigated EGFR-TKI sensitive mutations included G719C, G719S, G719A, L858R, L861Q, and exon 19 deletions, as well as a gefitinib-resistant mutation, T790M.

2.2. Statistical Analysis. Differences among the categorized groups were compared using either the two-sided chi-square test or Fisher's exact test. The survival was estimated by the Kaplan-Meier method, and differences in survival between the subgroups were analyzed by the log rank test. Data were analyzed using the StatView version 5.0J software package (Statistical Analysis Systems, Cary, NC, USA).

\section{Results}

3.1. Subtypes of EGFR Mutations. We examined the EGFR mutation status in 202 patients between April 2008 and November 2010. We excluded 4 patients from this study for the following reasons: one had small cell lung cancer, two had gastric cancer, and one had parotid cancer. Of the 198 patients with nonsmall cell lung cancer, 52 patients (26.3\%) had EGFR-TKI-sensitive EGFR mutations, and one patient had an EGFR-TKI-resistant mutation (T790M) with an EGFR-TKI-sensitive mutation (Exon 19 deletion). The patient population in this analysis (Table 1) was a little young, including more female, less never-smoker, and less squamous cell carcinoma of the lung in comparison with the lung cancer cohort that we previously published [12].

3.2. The Variables Associated with the EGFR Mutation Status. We investigated the association of several variables with the EGFR mutations (Table 2). A two-sided chi-square test showed that gender (female), smoking status (never smoker), histology (adenocarcinoma), and chest CT findings (normal) were significantly associated with the presence of an EGFR mutation. Of 122 patients with normal lungs, 69 patients had no history of smoking and 53 patients had a history of smoking. The frequency of EGFR mutations $(n, \%)$ in patients with normal lungs did not differ between smokers $(17,32.1 \%)$ and never-smokers $(26,37.7 \%)(P=0.5698)$.

3.3. Prognosis of Patients with EGFR Mutations Treated with Gefitinib. All patients with an EGFR mutation were treated in the Division of Respirology and Chest Surgery, NTT Medical Center Tokyo. Of the 52 patients with EGFR mutation, 43 patients received gefitinib. The clinical characteristics of the patients with an EGFR mutation treated with gefitinib are shown in Table 3. The median survival after gefitinib treatment was 797 days. We identified ILD in two patients during gefitinib treatment; one had no ILD before gefitinib treatment and one had pulmonary fibrosis. The patient with pulmonary fibrosis developed acute exacerbation of preexisting ILD on day 7 of gefitinib treatment and died on day 14 because of ILD. The survival curves of the 42 patients, excluding the patient with pulmonary fibrosis, according to smoking status and chest CT results, are shown in Figures $1(\mathrm{a})$ and $1(\mathrm{~b})$, respectively. No differences in survival were observed between smokers $(n=18$, MST not reached) and never-smokers $(n=24$, MST 797 days $)$ or between 
TABLE 1: Patient characteristics NSCLC: nonsmall cell lung cancer: LCNEC; large cell neuroendocrine carcinoma.

\begin{tabular}{|c|c|}
\hline Total number of patients & 198 \\
\hline Age (median, range) & $68,28-92$ \\
\hline \multicolumn{2}{|l|}{ Gender } \\
\hline Female & 86 \\
\hline Male & 112 \\
\hline \multicolumn{2}{|l|}{ Smoking-status } \\
\hline Never & 74 \\
\hline Ex/Current & 124 \\
\hline \multicolumn{2}{|l|}{ Histology } \\
\hline Adenocarcinoma & 169 \\
\hline Squamous cell carcinoma & 9 \\
\hline Other NSCLC & 15 \\
\hline LCNEC & 4 \\
\hline \multicolumn{2}{|l|}{ Clinical stage of NSCLC } \\
\hline IA & 29 \\
\hline IB & 14 \\
\hline IIA & 2 \\
\hline IIB & 6 \\
\hline IIIA & 12 \\
\hline IIIB & 30 \\
\hline IV & 105 \\
\hline \multicolumn{2}{|l|}{ Chest CT } \\
\hline Normal & 122 \\
\hline Emphysema & 59 \\
\hline Fibrosis & 17 \\
\hline \multicolumn{2}{|l|}{ EGFR mutation } \\
\hline Wild type & 147 \\
\hline Ex18 G718S & 1 \\
\hline Ex19 del & 34 \\
\hline Ex21 L858R & 15 \\
\hline EX19 del + Ex21 L858R & 1 \\
\hline Ex 19del + T790M & 1 \\
\hline
\end{tabular}

patients with normal lung ( $n=36$, MST 874 days) and those with emphysematous lungs ( $n=6$, MST 749 days) on chest CT.

\section{Discussion}

We herein showed the frequency of EGFR mutation in nonsmall cell lung cancer to be high in patients with the following factors: female gender, no history of smoking, adenocarcinoma, and normal lungs on chest CT. A survival analysis of the patients with EGFR mutations, excluding one patient with pulmonary fibrosis, showed no differences between smokers and never-smokers or between patients with emphysema and those with normal lungs on chest CT.

There is considerable variability in the susceptibility of smokers to developing smoking-related pulmonary diseases [14-16]. The incidence of lung cancer is increased in patients with emphysema and fibrosis, and this effect is independent
TABLE 2: Patient characteristics and EGFR mutation status.

\begin{tabular}{lccc}
\hline & Number & $\begin{array}{c}\text { EGFR mutation } \\
(n, \%)\end{array}$ & $P$-value \\
\hline Gender & & & \\
$\quad$ Male & 112 & $17,15.2 \%$ & $P<0.0001$ \\
$\quad$ Female & 86 & $35,40.7 \%$ & \\
Age & & & \\
$\quad<65$ & 80 & $23,28.8 \%$ & $P=0.5156$ \\
$\quad 65 \leq$ & 118 & $29,24.6 \%$ & \\
Histology & & & \\
$\quad$ Adenocarcinoma & 169 & $50,29.6 \%$ & $P=0.0107$ \\
$\quad$ Nonadenocarcinoma & 29 & $2,6.9 \%$ & \\
Smoking status & & & \\
$\quad$ Never & 74 & $29,39.2 \%$ & $P=0.0139$ \\
Ex/Current & 124 & $23,18.5 \%$ & \\
Clinical stage of NSCLC & & & \\
I-IIIA & 63 & $21,33.3 \%$ & $P=0.1649$ \\
IIIB-IV & 135 & $31,22.9 \%$ & \\
Chest CT & & & \\
Normal & 122 & $43,35.2 \%$ & \\
Emphysema & 59 & $8,13.6 \%$ & $P=0.0011$ \\
Fibrosis & 17 & $1,5.8 \%$ & \\
\hline
\end{tabular}

of the effect of cigarette smoking $[17,18]$. We consider that smokers with emphysema or fibrosis are more susceptible to smoking-related inflammation compared to those with normal lungs. Although the frequency of EGFR mutation was low in patients with emphysema and fibrosis, the frequency in those with normal lungs was not different between smokers and never-smokers. Our data suggested that smokers with normal lungs were not susceptible to smoking-related inflammation, and that nonsmall cell lung cancer in smokers with normal lungs showed the same biological features to that in never-smokers. Further investigations are necessary to elucidate whether smokingrelated pulmonary diseases and lung cancer might result from overlapping or associated genetic variants implicated in smoking-related inflammation.

Although a history of smoking and the coexistence of emphysema were negatively associated with the frequency of EGFR mutations, these clinical factors did not affect the prognosis of the patients with EGFR mutations treated with gefitinib. Toyooka et al. showed that epidermal growth factor receptor mutation, but not sex or smoking, is independently associated with a favorable prognosis of gefitinibtreated patients with lung adenocarcinoma [5]. EGFRTKI treatment should be considered in patients with an EGFR mutation, even if they have a history of smoking or emphysema without fibrosis.

The presence of EGFR mutations in patients with pulmonary fibrosis was rare in this study. Only one (5.9\%) of 17 patients with pulmonary fibrosis had an EGFR mutation. Preexisting chronic ILD is known as a risk factor for ILD in gefitinib treatment [10]. In this study, one patient with 
TABLE 3: Characteristics of patients with an EGFR mutation treated with gefitinib.

\begin{tabular}{lc}
\hline Total number & 43 \\
\hline Age (median, range) & $67,28-92$ \\
Gender & \\
Male & 13 \\
Female & 30 \\
Smoking-status & \\
Never & 24 \\
Ex/Current & 19 \\
Pack-years of smokers (median, range) & $2.5-225$ \\
Histology & \\
Adenocarcinoma & 42 \\
Squamous cell carcinoma & 1 \\
Clinical stage of NSCLC & \\
IB & 2 \\
IIIA & 1 \\
IIIB & 7 \\
IV & 22 \\
Recurrence & 11 \\
History of chemotherapy before gefitinib treatment & \\
No & 28 \\
Yes & 15 \\
EGFR mutation & 10 \\
Ex18 G719C & 1 \\
Ex19 del & \\
Ex21 L858R & 30 \\
Ex19 del + Ex21L858R & \\
Ex19 del + Ex20 T790M & 1 \\
Chest CT & \\
Normal & \\
Emphysema & \\
Fibrosis & \\
\hline
\end{tabular}

pulmonary fibrosis and an EGFR mutation treated with gefitinib developed fatal ILD.

The present study had several limitations, including the fact that it was observational and uncontrolled in design and was performed at a single institution, with retrospective collection of data. The results may have been subject to some selection and treatment bias. The indications for therapy and the selection of treatment were not uniform for all patients, thereby limiting the evaluation of the effects of treatment. The data presented herein should not be interpreted as providing an appropriate evaluation of the efficacy of treatment, which will require randomized prospective studies. A multivariate analysis could not be performed due to the small sample size, and it was therefore not possible to evaluate the potential confounding effects of various other variables related to survival. However, the existence of emphysema and fibrosis on chest CT were prospectively identified at the diagnosis of lung cancer. The EGFR mutation status was identified before the EGFR-TKI treatment. Data on the

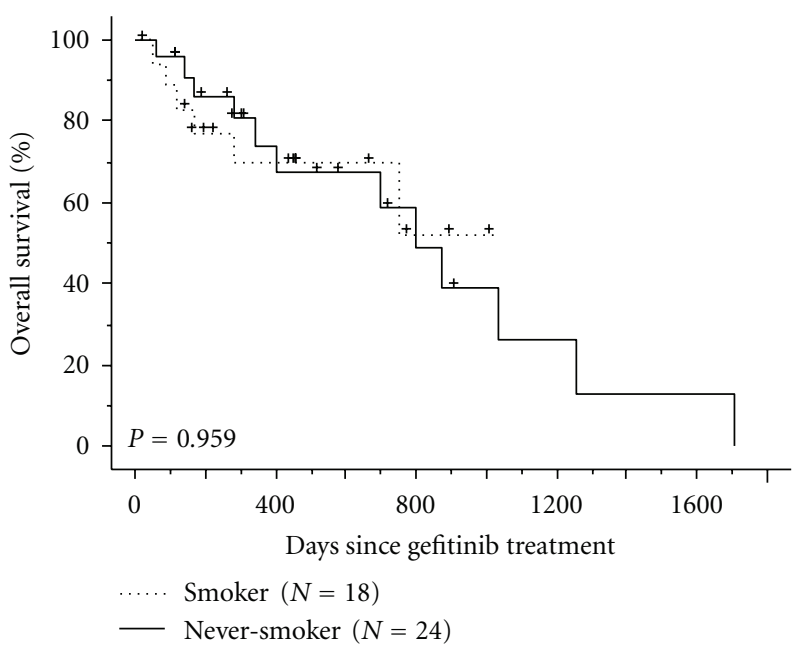

(a)

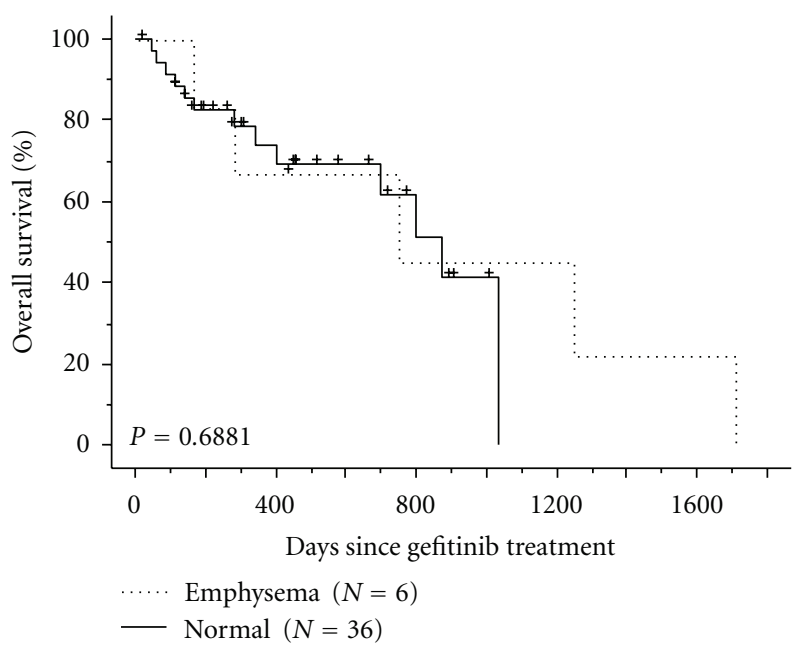

(b)

FIGURE 1: (a) Overall survival of patients with an EGFR mutation treated with gefitinib, according to smoking status (never smokers: solid line; smokers: dotted line). +: censored patient. (b) Overall survival of patients with an EGFR mutation treated with gefitinib, according to underlying pulmonary disease (normal: solid line; emphysema: dotted line). +: censored patient.

demographic characteristics and survival of patients were unlikely to be affected by the study design.

In summary, the frequency of EGFR mutations in patients with normal lungs on chest CT was not different between smokers and never-smokers. Of patients with sensitive EGFR mutations and normal lungs on chest CT, smokers had a comparable prognosis with never-smokers. Selecting patients on the basis of chest CT, rather than the smoking status, would likely result in a population with a greater sensitivity to gefitinib.

\section{Conflict of Interests}

The authors declare that there is no conflict of interests. 


\section{References}

[1] M. Fukuoka, S. Yano, G. Giaccone et al., "Multi-institutional randomized phase II trial of gefitinib for previously treated patients with advanced non-small-cell lung cancer," Journal of Clinical Oncology, vol. 21, no. 12, pp. 2237-2246, 2003, Erratum in: Journal of Clinical Oncology, vol. 22, no. 23, pp. 4811, 2004.

[2] T. J. Lynch, D. W. Bell, R. Sordella et al., "Activating mutations in the epidermal growth factor receptor underlying responsiveness of non-small-cell lung cancer to gefitinib," New England Journal of Medicine, vol. 350, no. 21, pp. 2129-2139, 2004.

[3] J. G. Paez, P. A. Jänne, J. C. Lee et al., "EGFR mutations in lung, cancer: correlation with clinical response to gefitinib therapy," Science, vol. 304, no. 5676, pp. 1497-1500, 2004.

[4] T. Mitsudomi, T. Kosaka, H. Endoh et al., "Mutations of the epidermal growth factor receptor gene predict prolonged survival after gefitinib treatment in patients with non-smallcell lung cancer with postoperative recurrence," Journal of Clinical Oncology, vol. 23, no. 11, pp. 2513-2520, 2005.

[5] S. Toyooka, T. Takano, T. Kosaka et al., "Epidermal growth factor receptor mutation, but not sex and smoking, is independently associated with favorable prognosis of gefitinib-treated patients with lung adenocarcinoma," Cancer Science, vol. 99, no. 2, pp. 303-308, 2008.

[6] T. Mitsudomi, S. Morita, Y. Yatabe et al., "Gefitinib versus cisplatin plus docetaxel in patients with non-small-cell lung cancer harbouring mutations of the epidermal growth factor receptor (WJTOG3405): an open label, randomised phase 3 trial," The Lancet Oncology, vol. 11, no. 2, pp. 121-128, 2010.

[7] M. Maemondo, A. Inoue, K. Kobayashi et al., "Gefitinib or chemotherapy for non-small-cell lung cancer with mutated EGFR," New England Journal of Medicine, vol. 362, no. 25, pp. 2380-2388, 2010.

[8] M. H. Cohen, G. A. Williams, R. Sridhara, G. Chen, and R. Pazdur, "FDA drug approval summary: gefitinib (ZD1839) (Iressa ${ }^{\circledR}$ ) tablets," Oncologist, vol. 8, no. 4, pp. 303-306, 2003.

[9] M. Ando, I. Okamoto, N. Yamamoto et al., "Predictive factors for interstitial lung disease, antitumor response, and survival in non-small-cell lung cancer patients treated with gefitinib," Journal of Clinical Oncology, vol. 24, no. 16, pp. 2549-2556, 2006.

[10] S. Kudoh, H. Kato, Y. Nishiwaki et al., "Interstitial lung disease in Japanese patients with lung cancer: a cohort and nested case-control study," American Journal of Respiratory and Critical Care Medicine, vol. 177, no. 12, pp. 1348-1357, 2008.

[11] V. Cottin, H. Nunes, P. Y. Brillet et al., "Combined pulmonary fibrosis and emphysema: a distinct underrecognised entity," European Respiratory Journal, vol. 26, no. 4, pp. 586-593, 2005.

[12] K. Usui, C. Tanai, Y. Tanaka, H. Noda, and T. Ishihara, "The prevalence of pulmonary fibrosis combined with emphysema in patients with lung cancer," Respirology, vol. 16, no. 2, pp. 326-331, 2011.

[13] A. Sutani, Y. Nagai, K. Udagawa et al., "Gefitinib for nonsmall-cell lung cancer patients with epidermal growth factor receptor gene mutations screened by peptide nucleic acidlocked nucleic acid PCR clamp," British Journal of Cancer, vol. 95, no. 11, pp. 1483-1489, 2006.

[14] R. J. Hung, J. D. McKay, V. Gaborieau et al., "A susceptibility locus for lung cancer maps to nicotinic acetylcholine receptor subunit genes on 15q25," Nature, vol. 452, no. 7187, pp. 633-637, 2008.
[15] T. E. Thorgeirsson, F. Geller, P. Sulem et al., "A variant associated with nicotine dependence, lung cancer and peripheral arterial disease," Nature, vol. 452, no. 7187, pp. 638-642, 2008.

[16] D. Lambrechts, I. Buysschaert, P. Zanen et al., “The 15q24/25 susceptibility variant for lung cancer and chronic obstructive pulmonary disease is associated with emphysema," American Journal of Respiratory and Critical Care Medicine, vol. 181, no. 5, pp. 486-493, 2010.

[17] R. P. Young, R. J. Hopkins, T. Christmas, P. N. Black, P. Metcalf, and G. D. Gamble, "COPD prevalence is increased in lung cancer, independent of age, sex and smoking history," European Respiratory Journal, vol. 34, no. 2, pp. 380-386, 2008.

[18] R. Hubbard, A. Venn, S. Lewis, and J. Britton, "Lung cancer and cryptogenic fibrosing alveolitis: a population-based cohort study," American Journal of Respiratory and Critical Care Medicine, vol. 161, no. 1, pp. 5-8, 2000. 


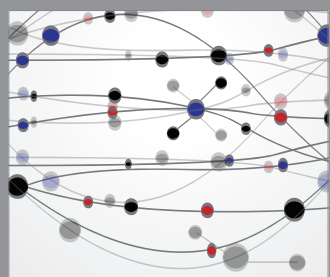

The Scientific World Journal
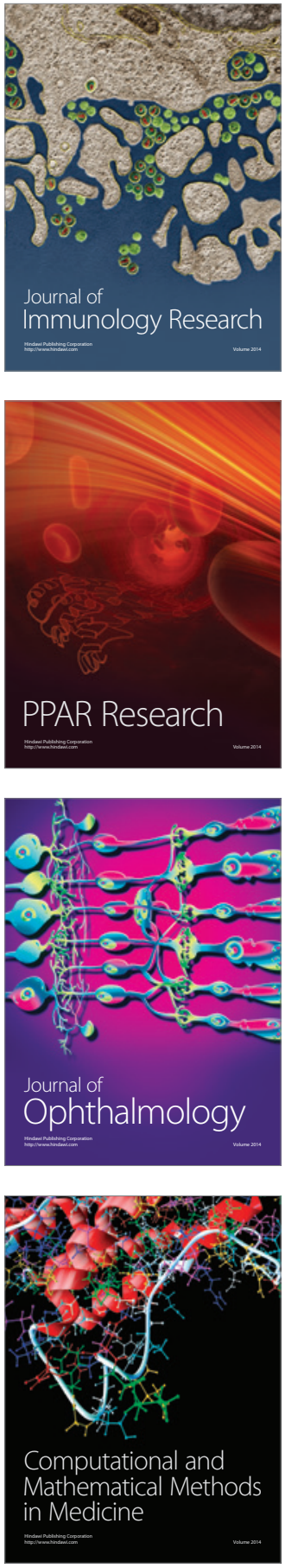

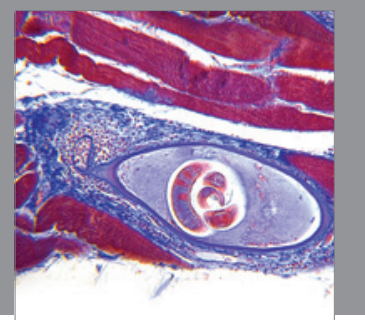

Gastroenterology

Research and Practice
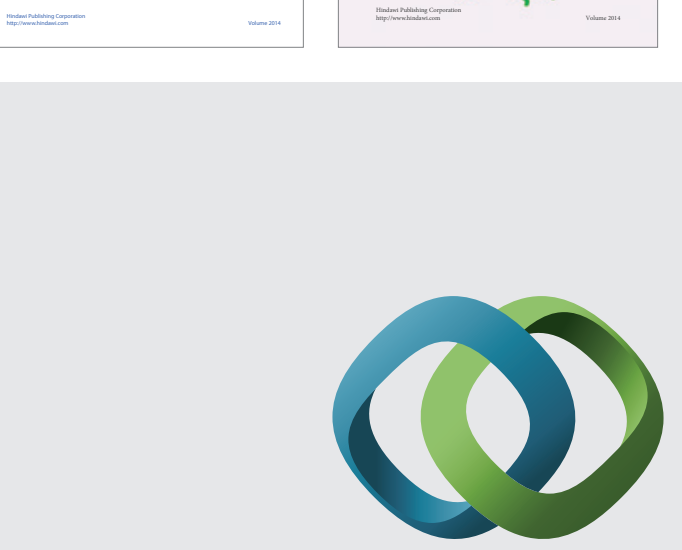

\section{Hindawi}

Submit your manuscripts at

http://www.hindawi.com
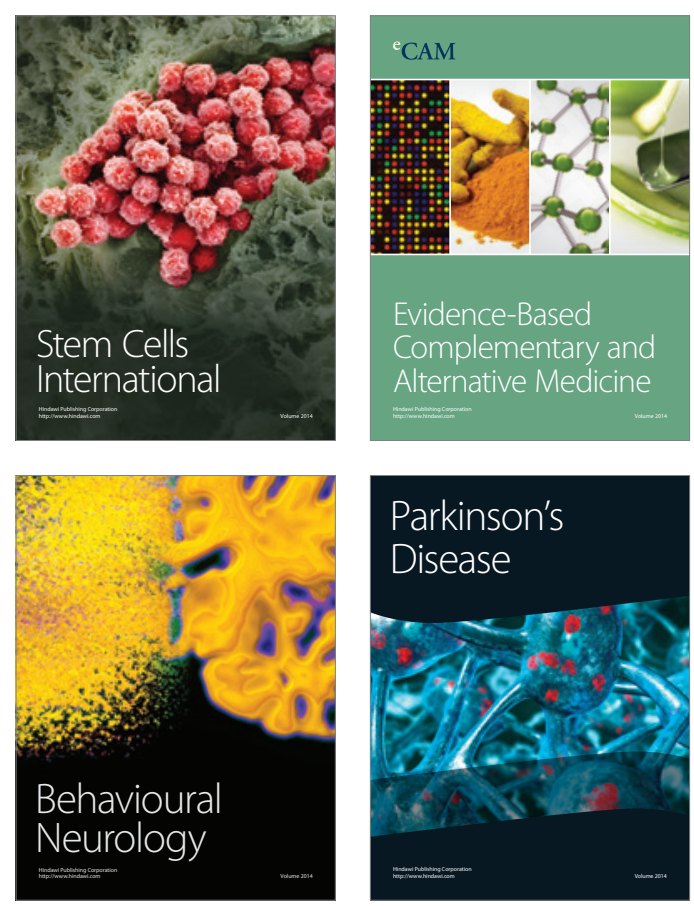

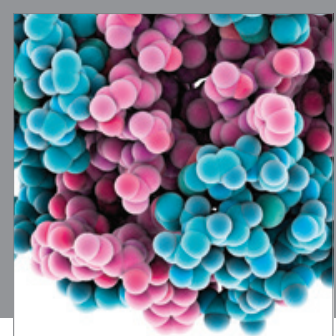

Journal of
Diabetes Research

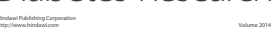

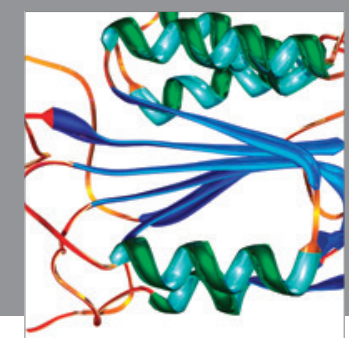

Disease Markers
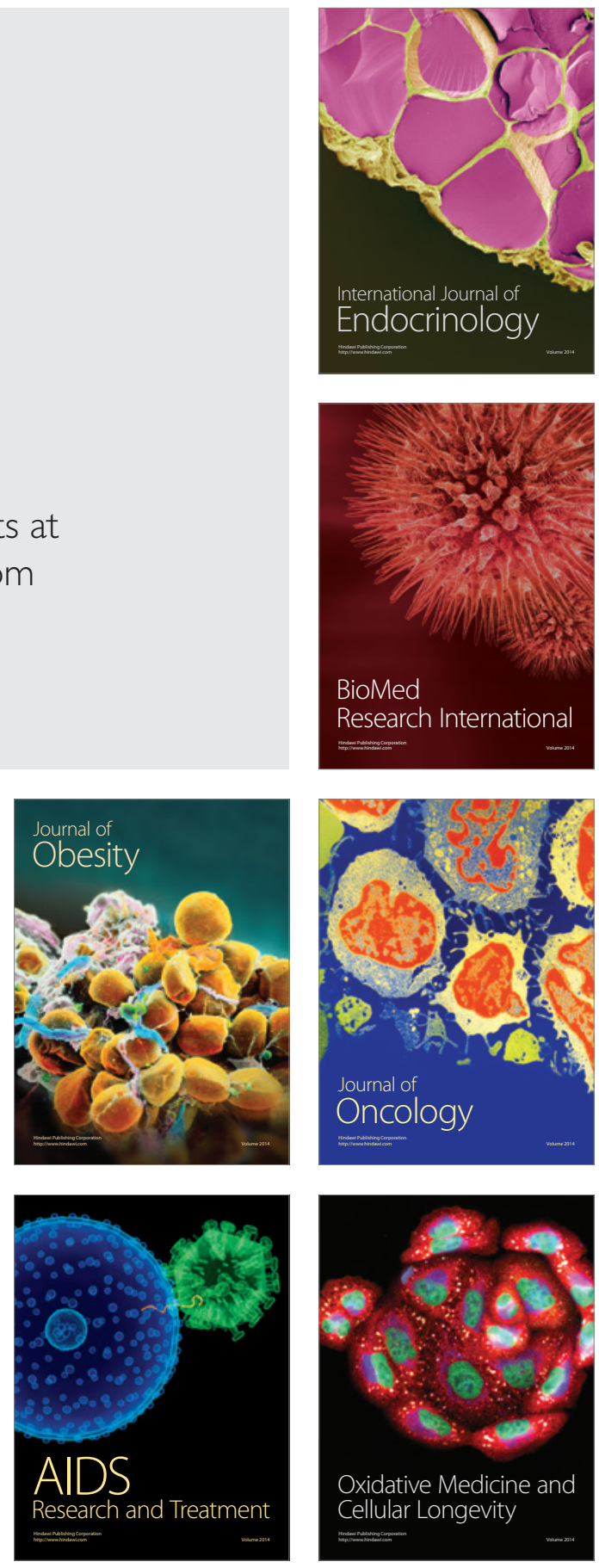